\title{
Carcinoma hepatocelular
}

\section{Algoritmo diagnóstico y terapéutico del carcinoma hepatocelular}

\author{
A. Linares, M. Rodríguez, L. Rodrigo
}

El carcinoma hepatocelular $(\mathrm{CHC})$ es la principal neoplasia maligna del hígado. Aumentó su incidencia en los últimos años, hasta el punto de ser actualmente el quinto tumor más frecuente en el mundo, salvo excepciones en países del Sudeste Asiático y Africa, donde puede aparecer en portadores del virus de la hepatitis B sin hepatopatía avanzada. Ocurre en pacientes con cirrosis hepática, en los que constituye la principal causa de muerte ${ }^{1,2}$. La incidencia ajustada para la edad en los países del sur de Europa se sitúa en 10 casos por 100.000 habitantes varones. No todos los pacientes con cirrosis tienen el mismo riesgo de complicarse con un $\mathrm{CHC}$. Nosotros hemos diseñado un modelo basado en variables fáciles de determinar, como son la infección por virus $C$, la edad, la cifra de plaquetas y la tasa de protrombina, que permite seleccionar un grupo con riesgo elevado de desarrolar $\mathrm{CHC}^{3}$.

\section{Diagnóstico}

Se puede detectar una lesión focal en el hígado en dos contextos clínicos muy distintos.

1) Cuando se evalúa por primera vez un paciente sin enfermedad hepática conocida, que acude a la consulta ambulatoria o ingresa por una complicación (ictericia, ascitis, encefalopatía hepática, hemorragia digestiva) o por agravamiento del estado general.

2) En un paciente con cirrosis hepática conocida al que se revisa de forma periódica.

En el primer caso, es frecuente que los pacientes presenten un tumor evolucionado y no sean candidatos a tratamiento curativo, mientras que en el segundo, aunque se carece de estudios controlados, se diagnostica hasta un 30$40 \%$ de pacientes subsidiarios de tratamientos curativos, por

Servicio de Aparato Digestivo

Hospital Universitario Central de Asturias lo que la opinión generalizada es la de realizar vigilancia a los pacientes con cirrosis hepática para la detección precoz de un $\mathrm{CHC}^{4}$.

Los programas de vigilancia periódica o "screening" para el diagnóstico precoz del carcinoma hepatocelular

No se conoce hasta la fecha cual es el procedimiento ideal de vigilancia periódica para el diagnóstico precoz del CHC. Las cuestiones pendientes más importantes son:

1) Si se debe aplicar a todos los pacientes con cirrosis o sólo a aquellos en los que ya se ha establecido un mayor riesgo:

2) La periodicidad de las evaluaciones;

3) Las pruebas diagnósticas óptimas y

4) La eficiencia de este proceder en términos coste-beneficio, lo que debería traducirse en una mayor probabilidad de ser sometidos a un tratamiento curativo y en última instancia en una mayor supervivencia.

En líneas generales, el programa de vigilancia se debe emplear en aquellos pacientes con cirrosis hepática en los que en el caso de objetivar un $\mathrm{CHC}$ serían candidatos a un tratamiento curativo, como son los que no tienen una edad avanzada, la función hepática preservada, ausencia de enfermedades graves que resultasen en contraindicación para la resección o el trasplante, ausencia de alcoholismo activo, y adecuado cumplimiento terapéutico. No se plantea en pacientes con la función hepática muy alterada porque en ellos habría que considerar el trasplante al margen de la existencia, asociada o no, de un CHC. En cuanto a la periodicidad de la vigilancia, los datos actuales sugieren que el tiempo que transcurre desde una lesión no detectable hasta que ésta mida $2 \mathrm{~cm}$, oscila entre 4 y 12 meses ${ }^{5}$ y por ello, con el objeto de detectar un tumor menor de $3 \mathrm{~cm}$ de diámetro, el intervalo más idóneo sugerido es el semestral, en los pacientes de riesgo. En aquellos en los que el riesgo es muy bajo, sería suficiente un seguimiento clínico con una periodicidad no determinada (por ejemplo anual), con el objeto de evaluar si pasan a ser de riesgo elevado. Las pruebas que se utilizan en la mayoría de los casos son la determinación de alfa-fetoproteína y la ecografía. Ambas son baratas y de fácil realización; sin embargo, la sensibilidad de las mismas es baja. La alfa-fetoproteína tiene una sensibilidad de tan sólo el 39$67 \%$, con una especificidad entre el 76 y el $91 \%$ dependien- 


\section{A. Linares y cols.}

do del valor de corte que se considere ${ }^{6}$ y la ecografía presenta una tasa muy elevada de falsos negativos, (sensibilidad $71 \%$, especificidad $93 \%)^{4}$ que depende además de las limitaciones propias de la técnica, a que es una exploración muy dependiente del observador y a que se sospecha que en la evaluación clínica rutinaria el rendimiento es sustancialmente inferior al que se objetiva en los estudios de investigación. Por ello se contempla el empleo de la tomografía computerizada como alternativa, pero este procedimiento es mucho más costoso, si bien no disponemos hasta la fecha de estudios controlados.

\section{Evaluación de una lesión hepática focal en un hígado con cirrosis hepática}

Cuando mediante un procedimiento de vigilancia periódica o bien de forma casual, se detecta una lesión hepática focal, es esencial tener en cuenta su tamaño, así como la existencia o no de cirrosis hepática, para decidir el procedimiento diagnóstico más idóneo que se debe realizar. Tal como mostramos en la Fig. 1, las lesiones iguales o menores de $1 \mathrm{~cm}$ no suelen ser malignas, mientras que entre 1 y $2 \mathrm{~cm}$. y las mayores, tienen una elevada probabilidad de ser malignas. En la Tabla I mostramos los criterios diagnósticos.

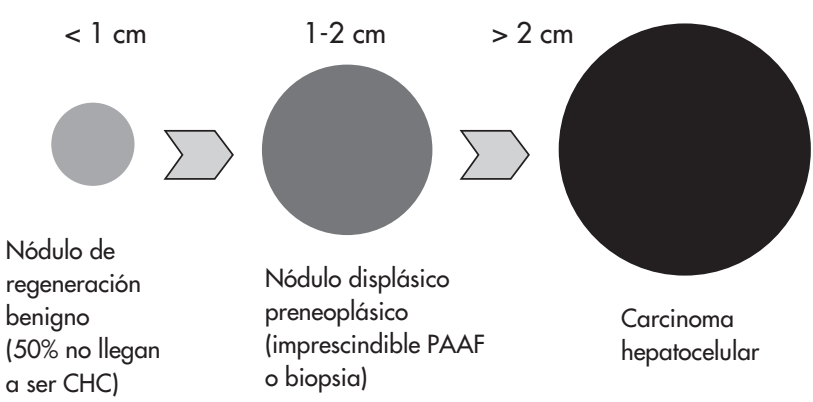

Fig. 1. Significado de las lesiones focales en el hígado cirrótico.

TABLA I

\section{Criterios diagnósticos de CHC}

1. Criterio cito-histológico. Es obligado si la lesión es igual o menor de $2 \mathrm{~cm}$ y la alfa-fetoproteína igual o menor de $400 \mathrm{ng} / \mathrm{ml}$.

2. Criterios no invasivos. Aplicables sólo cuando se trata de pacientes cirróticos.

a. Criterio radiológico: Lesión focal $>$ de $2 \mathrm{~cm}$ con dos técnicas de imagen' coincidentes demostrando hipervascularización arterial.

b. Criterio combinado: Lesión focal $>$ de $2 \mathrm{~cm}$ con hipervascularización arterial asociada a niveles de alfa-fetoproteína $>400 \mathrm{ng} / \mathrm{ml}$

' Técnicas de imagen: ecografía, TC,RMN, arteriografía

La demostración de una lesión focal en el hígado resuelve sólo parte del problema, puesto que no todos los nódulos que aparecen en un hígado cirrótico son malignos. Tal como mos- tramos en la Fig. 1, pueden existir nódulos de regeneración grandes, que habitualmente miden hasta $1 \mathrm{~cm}$. y el seguimiento de los mismos demuestra que hasta un $50 \%$ de las veces siguen siendo benignos. En segundo lugar pueden existir nódulos displásicos preneoplásicos, que en su mayoría miden entre 1 y $2 \mathrm{~cm}$ y cuya naturaleza es preciso aclarar, bien mediante técnicas de imagen fiables o bien mediante histología. Los nódulos superiores a $2 \mathrm{~cm}$ suelen ser malignos y para su diagnóstico se considera suficiente la realización de técnicas no invasivas, en la mayoría de los casos. Finalmente, existen en ocasiones lesiones hipervasculares benignas, en su mayoría angiomas, que nada tienen que ver con la cirrosis y que son hallazgos frecuentes, al igual que en hígados sanos.

El algoritmo diagnóstico se muestra en la Fig. 2. Dicho algoritmo fue consensuado por un grupo de expertos en el año $2000^{2}$. Toda lesión igual o mayor de $1 \mathrm{~cm}$ tiene muchas probabilidades de ser un $\mathrm{CHC}$ o de acabar siéndolo, aunque no experimente cambios de tamaño en controles sucesivos y el comportamiento dinámico en la TC y la RM no sea típica de un $\mathrm{CHC}$, e incluso aunque la PAAF o la biopsia dirigida con control ecográfico sean negativas.

\section{Evaluación de la extensión}

Del rigor en la evaluación de la extensión dependen las opciones terapéuticas. Si se trata de un paciente al que en el estudio ecográfico se encuentra un tumor y en el que debido al estadio avanzado no se plantea ninguna opción terapéuti$\mathrm{ca}$, no es preciso realizar estudios adicionales. En cambio, cuando se plantea un tratamiento curativo o paliativo, es imprescindible conocer bien el tamaño del nódulo o nódulos, precisar con exactitud su número, la existencia de invasión vascular macroscópica y en el caso de que el paciente vaya a ser sometido a cirugía o a trasplante hepático, descartar afectación pulmonar y ósea. Hasta la fecha, la experiencia demuestra que casi todos los procedimientos de imagen infraestiman la extensión de la enfermedad cuando se comparan con el patrón oro, que es el estudio detallado del explante de un paciente sometido a trasplante hepático. A medida que los procedimientos de imagen son más sensibles, va siendo posible aproximarse, cada vez más a la realidad, sobre todo en cuanto a la presencia de pequeños nódulos satélites y en cuanto a la invasión vascular. Se estima que con las técnicas de TC y resonancia convencionales, que compiten a medida que se suceden los avances tecnológicos en cada una de las técnicas, se omiten entre un 30-50\% de nódulos, la mayoría menores de $2 \mathrm{~cm}^{8-10}$.

Con estos equipos la angiografía no suele aportar nada y ha dejado de hacerse de rutina. La TC, previa administración de lipiodol, también se ha desechado. La ecografía es poco útil para precisar la existencia de nódulos adicionales, sobre todo si son menores de $2 \mathrm{~cm}^{7}$. Los equipos que incorporan Doppler, angio-Doppler, segundo armónico y pulsoinvertido son, de todos modos, de gran ayuda para valorar la permeabilidad vascular. Factor esencial para tomar decisiones terapéuticas, el empleo de ecopotenciadores incrementa la sensibilidad diagnóstica y permite valorar la necrosis después del tratamiento percutáneo, aunque es una técnica menos sensible que la TC. La angio-TC y la angio-resonancia se emplean indistintamente, prácticamente siempre precedidas de una ecografía-Doppler para valorar la permeabilidad vascular y las anomalías anatómicas arteriales en 


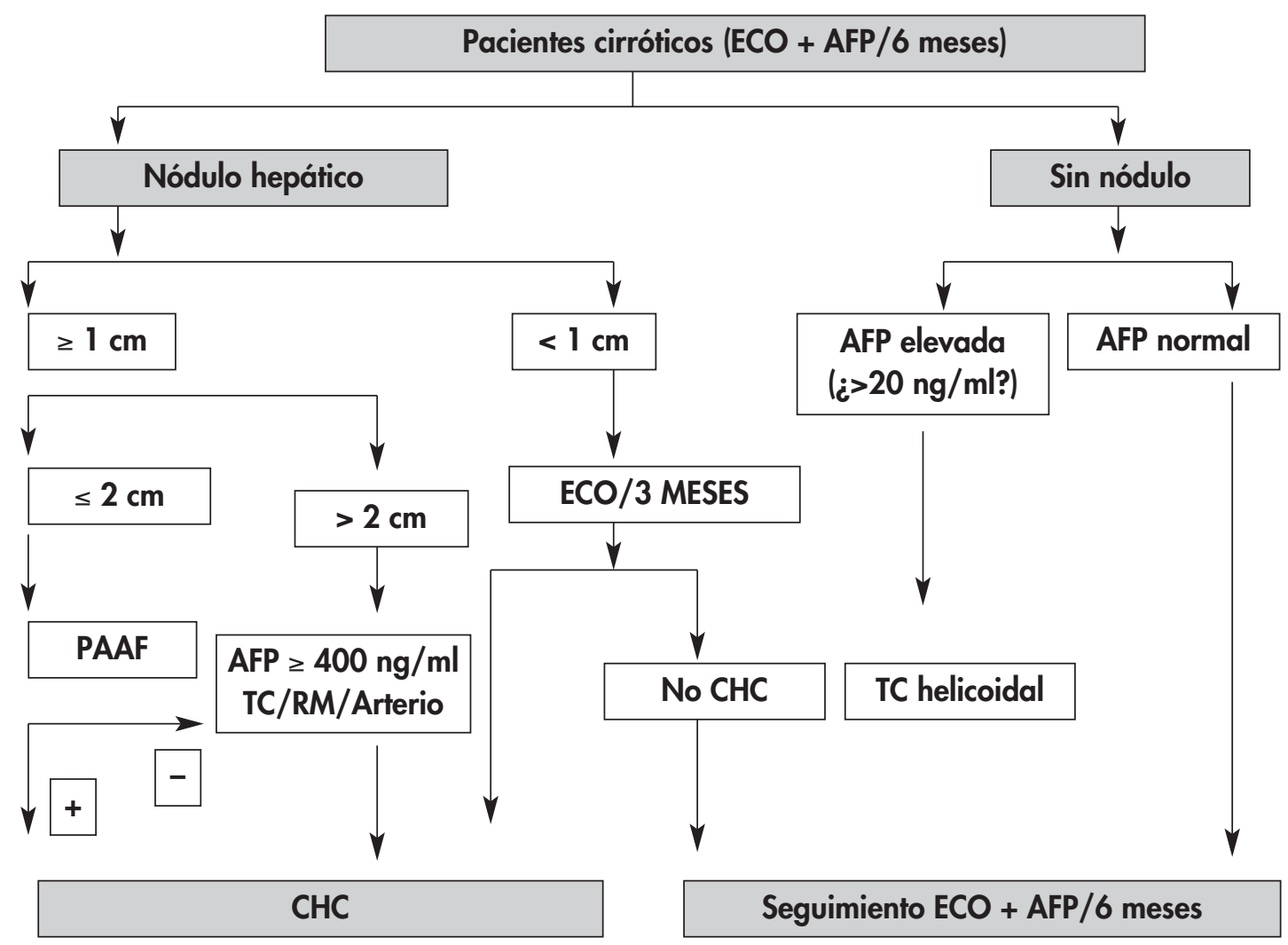

Fig. 2. Algoritmo diagnóstico del carcinoma hepatocelular.

los pacientes que van a ser sometidos a resección y fundamentalmente a trasplante hepático, pero no son de utilidad diagnóstica, y rara vez se emplea la arteriografía como procedimiento diagnóstico, reservándose para situaciones en los que la angio-TC o la angio-resonancia plantean dudas diagnósticas o para valorar la presencia de nódulos hipervasculares, para realizar embolización o quimioembolización.

Los estudios actuales parecen otorgar mayor protagonismo a las técnicas de resonancia de última generación, en detrimento de las técnicas basadas en la TC trifásica ${ }^{11}$.

La dificultad diagnóstica se hace más patente cuando un paciente con cirrosis hepática presenta más de un nódulo. La confirmación de un $\mathrm{CHC}$ en uno de ellos, habitualmente el de mayor tamaño, no presupone que la naturaleza de los restantes nódulos sea también maligna. Puede tratarse de nódulos displásicos o de nódulos de regeneración. En un estudio realizado en el Hospital Clínico ${ }^{11}$ en 29 explantes de pacientes trasplantados por $\mathrm{CHC}, 76$ nódulos eran $\mathrm{CHC}$, pero 51 nódulos adicionales no lo eran (13 nódulos displásicos, 31 nódulos de regeneración y 7 hemangiomas). Dado que el número de lesiones malignas es determinante para decidir un tratamiento curativo o meramente paliativo, es sumamente importante conocer las características de todas ellas. La sensibilidad de la RMN es del 100\% en los nódulos mayores de 20 mm, 89\% para los que miden 10-20 mm y sólo $34 \%$ para los menores de $10 \mathrm{~mm}$. Eso quiere decir que los nódulos pequeños, aunque sean un $\mathrm{CHC}$, no muestran el comportamiento típico. Por otra parte, en aquellos nódulos que se comportan como hipervasculares inespecíficos, y que miden más de $5 \mathrm{~mm}$, dos de cada tres han resultado ser también $\mathrm{CHC}$. En resumen, la sensibilidad de la RMN para valorar el número y las características de los nódulos es de un $76 \%$, la especificidad del $75 \%$ con una tasa de falsos positivos del 10\%, mientras que la sensibilidad de la TC trifásica es del 61\%, la especificidad se sitúa en tan sólo un $66 \%$ y la tasa de falsos positivos es ligeramente superior que la de la RMN (13\%) ${ }^{2}$. Las diferencias en cuanto a la sensibilidad se hacen más manifiestas si se trata de nódulos entre 10 y $20 \mathrm{~mm}$ ( $84 \%$ la RMN frente a $47 \%$ la TC). Sin embargo, ambas técnicas son muy poco útiles todavía para detectar $\mathrm{CHC}$ menores de $1 \mathrm{~cm}$ de diámetro, en los que además es especialmente necesaria la caracterización de los mismos, puesto que el comportamiento no es típico ${ }^{11}$.

Hasta la fecha, las decisiones terapéuticas más importantes, como la indicación de trasplante hepática, se basan en los criterios de Mazzaferro et a ${ }^{12}$. Mediante dichos criterios, basados en las técnicas de imagen convencionales, en las que no se diagnostica un número considerable de lesiones satélites, la recidiva tumoral se reduce a aproximadamente un $10 \%$. Sin embargo, al analizar la recidiva valorando el número y tamaño de los nódulos en el explante, se ha comprobado igualmente un buen pronóstico aún siendo los nódulos de mayor tamaño y mayor número que los considerados según los criterios de Mazzaferro ${ }^{13}$. Por ello, cuanto más se aproximen las técnicas de imagen a la realidad de lo que 
ocurre en el explante, más podremos precisar las indicaciones, permitiendo incluir pacientes que hasta ahora rechazábamos y excluir en cambio aquellos de aparentemente buen pronóstico porque no se detecta satelitosis ni invasión vascular mediante las técnicas convencionales, pero sí mediante las de última generación.

\section{Evaluación del pronóstico}

Dado que el CHC aparece en la mayor parte de los casos sobre una cirrosis hepática, el pronóstico depende en gran medida de la función hepática existente. Actualmente se consideran 4 factores decisivos que afectan al pronóstico:

1. El estadio, agresividad y patrón de crecimiento del tumor;

2. El estado general del paciente, definido habitualmente mediante el performance status (PST) de la OMS ${ }^{14}$;

3. La función hepática, medida mediante la escala de Child-Pugh ${ }^{15,16}$;

4. El tratamiento que se aplique.

No es fácil una clasificación pronóstica que tenga en cuenta todas las variables citadas. En la Tablas II-V mostramos las 4 clasificaciones más empleadas en la actualidad, a saber, la de Okuda ${ }^{17}$, la del grupo Italiano (CLIP Cancer of the Liver Italian Program Investigators) ${ }^{18,} 19$, la TNM modifica$\mathrm{da}^{13,20}$ y la de Barcelona ${ }^{21}$. La más empleada en nuestro medio es la clasificación de Barcelona, que a su vez tiene en cuenta la de Okuda, la funcionalidad de la OMS y la función hepática. Por una parte permite definir aceptablemente bien el pronóstico, pero su virtud fundamental es permitir definir de una forma objetiva el tratamiento a aplicar.

TABLA II

\begin{tabular}{lcccc}
\hline \multicolumn{4}{c}{${\text { Estadiaje de Okuda }{ }^{17}}$} & \\
\hline & I & II & III \\
\hline Tamaño tumoral $>50 \%$ & $(-)$ & 1 ó 2 (+) & 3 ó 4 (+) \\
Ascitis & $(-)$ & & \\
Albúmina $<3 \mathrm{~g} / \mathrm{dl}$ & $(-)$ & & \\
Bilirrubina $>3 \mathrm{mg} / \mathrm{dl}$ & $(-)$ & & \\
Supervivencia media & & & \\
$\quad$ sin tratamiento (meses) & 8,3 & 2,0 & 0,7 \\
\hline
\end{tabular}

\section{Tratamiento del carcinoma hepatocelular}

Los tratamientos se clasifican en curativos y paliativos. Aquellos con intención curativa son la resección, el trasplante hepático y los tratamientos percutáneos. El tratamiento paliativo más común es la quimioembolización, asociada a oclusión arterial siempre que ello sea posible. Para decidir el tratamiento idóneo, en nuestro medio nos guiamos por la clasificación de Barcelona (Fig. 3)².

\section{Tratamientos curativos}

El tratamiento quirúrgico, el trasplante y las técnicas percutáneas consiguen una tasa relativamente elevada de RC en
TABLA III

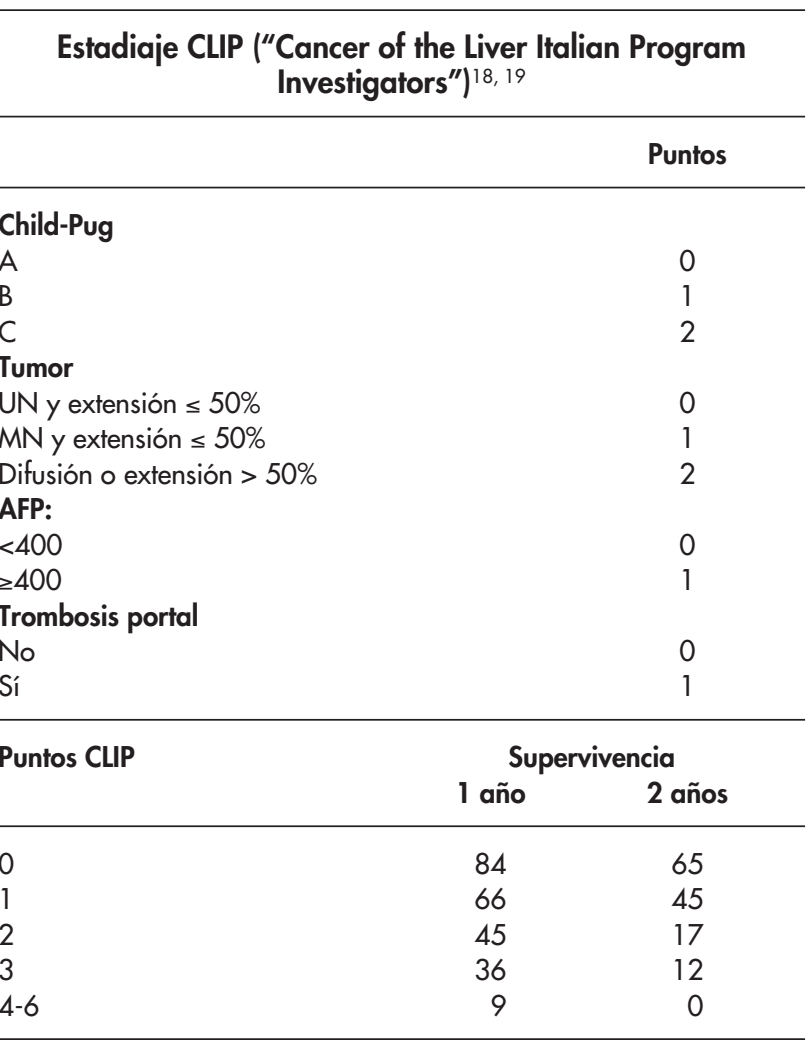

TABLA IV

Estadiaje TNM modificado ${ }^{13,20}$

\begin{tabular}{ll}
\hline T & Descripción \\
\hline T0 & No se objetiva tumor \\
T1 & Nódulo $\leq 1,9 \mathrm{~cm}$ \\
T2 & 1 nódulo $2-5 \mathrm{~cm}, 2 \circ 3$ nódulos todos \\
T3 & 1 nódulo $>5 \mathrm{~cm}, 2 \circ 3$ nódulos, al menos $1>3$ \\
& cm. \\
T4a & 4 o más nódulos, de cualquier tamaño \\
T4b & T2,T3 o T4 además de afectación portal intrahe- \\
& pática macroscópica o afectación de las venas su- \\
& prahepáticas, puesta de manifiesto con TAC, RM \\
& o Ecografía.
\end{tabular}

Estadio

$\begin{array}{ll}\text { Estadio I } & \text { T1 } \\ \text { Estadio II } & \text { T2 } \\ \text { Estadio III } & \text { T3 } \\ \text { Estadio IV A1 } & \text { T4a } \\ \text { Estadio IV A2 } & \text { T4b } \\ \text { Estadio IV B } & \text { Cualquier N1, cualquier M1 }\end{array}$

N1: Afectación de los ganglios regionales (hilio hepático) M1: Enfermedad metastásica, incluyendo la porta extrahepática o la afectación de las venas suprahepáticas. 
Estadiaje BCLC (Barcelona-Clinic Liver Cancer) $)^{21}$

\begin{tabular}{|c|c|c|c|c|c|c|}
\hline \multirow[b]{2}{*}{ Estadiaje CHC } & \multicolumn{2}{|c|}{ Status tumoral } & \multicolumn{2}{|c|}{ Status hepatopatía } & \multirow[b]{2}{*}{ Tratamiento } & \multirow[b]{2}{*}{ Supervivencia } \\
\hline & PST & Tumor & OKUDA & Status hepatopatía & & \\
\hline Estadio A: precoz & & & & & Radical & $50-70 \%$ a 5 años \\
\hline Al & 0 & Único & 1 & No htp y Bil normal & Cirugía & \\
\hline A2 & 0 & Único & I & Htp y Bil normal & Cirugía/Tx H & \\
\hline A3 & 0 & Único & I & htp y Bil elevada & Tx H/etanoliz & \\
\hline A4 & 0 & 3 nódulos $<3 \mathrm{~cm}$ & $|-| \mid$ & Child-Pugh A-B & $\mathrm{T} \times \mathrm{H}$ /etanoliz & \\
\hline Estadio B: Intermedio & 0 & Grande o multinodular & $|-| \mid$ & Child-Pugh A-B & Paliativo: TAE/TACE & $50 \%$ a 3 años \\
\hline Estadio C: Avanzado & $1-2$ & $\begin{array}{l}\text { Invasión vascular o } \\
\text { diseminación extrahepática }\end{array}$ & $|-I|$ & Child-Pugh A-B & Paliativo: Nuevas drogas & $50 \%$ a 6 meses \\
\hline Estadio D: Terminal & $3-4$ & Cualquiera & III & Child-Pugh C & Sintomático & $50 \%$ a 3 meses \\
\hline
\end{tabular}

Estadios A y B: todos los criterios deben cumplirse. Estadio C: al menos 1 criterio (PST 1-2 o invasión vascular/diseminación extrahepática). Estadio D: al menos 1 criterio (PST 3-4 o Okuda III/Child-Pugh C) PST: htp: hipertensión portal. Cirugía: resección quirúrgica. TxH: trasplante hepático. TP: tratamiento percutáneo (alcohol, radiofrecuencia) TAE/TACE: embolización transarterial o quimioembolización.

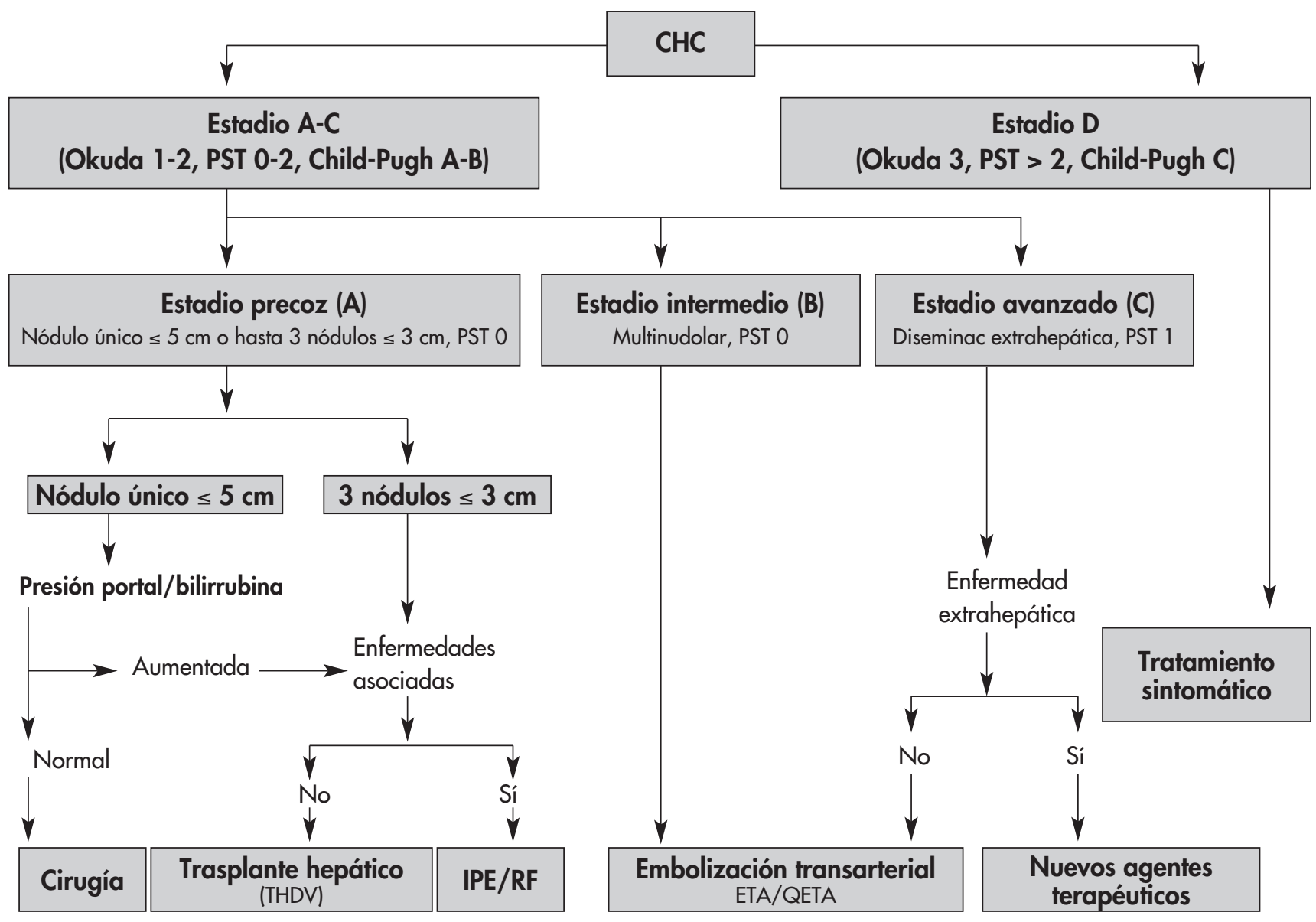

Fig. 3. Algoritmo terapéutico del CHC basado en la clasificación de Barcelona (BCLC) (2). IPE: Inyección percutánea de etanol; RF: Radiofrecuencia; ETA: embolización transarterial; QETA: quimioembolización transarterial; THDV: Trasplante hepático de donante vivo. 


\section{A. Linares y cols.}

los pacientes seleccionados, a saber, aquellos con un nódulo único menor de $5 \mathrm{~cm}$ o tres nódulos menores de $3 \mathrm{~cm}$. Hasta la fecha no se ha demostrado que en tumores más extendidos el tratamiento consiga una mayor supervivencia. No existen estudios aleatorizados controlados que comparen estas tres opciones.

El tratamiento quirúrgico proporciona resultados excelentes en pacientes con un nódulo único y una función hepática excelente; no es suficiente con un estadio Child Pugh A sino que la bilirrubina debe ser normal y no debe existir hipertensión portal ${ }^{22}$.

Las opciones de cirugía, de trasplante, tanto de cadáver como de donante vivo, así como de cirugía como puente para el trasplante, dependen de la lista de espera estimable, así como del grado de diferenciación, satelitosis e invasión vascular.

Los tratamientos percutáneos constituyen la mejor opción en pacientes con $\mathrm{CHC}$ inicial no tributarios de cirugía o de trasplante. Asimismo constituyen un tratamiento de los pacientes en lista de espera de trasplante hepático, cuando el tiempo en lista de espera es superior a 6 meses.

Entre los tratamientos percutáneos, el más empleado es la administración de alcohol absoluto; también se ha utilizado el ácido acético y está bastante difundida la radiofrecuencia.

La inyección intratumoral de alcohol (IIE) se tolera bien y es muy eficaz en tumores hasta $3 \mathrm{~cm}$ de diámetro, en donde se señala un $80 \%$ de respuestas completas ${ }^{23,24}$. En tumores entre 3 y $5 \mathrm{~cm}$ la tasa de respuesta completa (RC) se reduce a un $50 \%$ y es mucho menor en los mayores de $5 \mathrm{~cm}^{24,25}$, debido a infiltración incompleta en base a la gran cantidad de alcohol necesario o a la presencia de septos intratumorales que impiden la impregnación uniforme. Algunos estudios sugieren que la embolización seguida por la IIE mejora los resultados ${ }^{26}$.

La radiofrecuencia consigue una tasa de curaciones ligeramente superior a la del alcohol, con un menor número de sesiones, pero estos beneficios se contrarrestan por la mayor tasa de efectos adversos, un mayor riesgo de diseminación en el trayecto de la aguja y una menor aplicabilidad debido a la localización del tumor, puesto que en lesiones periféricas no se puede emplear ${ }^{27}$. Por otra parte, no se ha demostrado que sea superior a la inyección percutánea de alcohol absoluto ${ }^{28}$.

Los programas de vigilancia han supuesto la posibilidad de aplicar tratamientos curativos (resección, trasplante, IIE) a aproximadamente un $30-40 \%$ de los pacientes, con una supervivencia en su conjunto superior al $50 \%$ a los 5 años, pero ocurre recidiva entre un $60 \%$ y un $75 \%$ de los pacientes sometidos a tratamiento quirúrgico o a tratamientos percutáneos, $y$ en un 10 a un $25 \%$ de los pacientes sometidos a trasplante, a pesar de emplear criterios restrictivos $2,25,29,30$.

Cabe suponer, sobre el futuro de estas técnicas en la decisión, algo que ya sospechábamos debido a la elevada proporción de pacientes que recidivan de forma precoz con la cirugía y los tratamientos percutáneos: las nuevas modalidades terapéuticas inclinarán la balanza hacia el trasplante, al detectar nódulos adicionales malignos que previamente no eran diagnosticados con las pruebas de imagen convencionales.

\section{Tratamiento del $\mathrm{CHC}$ de pronóstico intermedio y avanzado}

Los pacientes en estadio B o C de la clasificación de Barcelona $(B C L C)$ presentan una supervivencia a los 3 años en- tre el 10 y el $50 \%^{2}$ y son candidatos a recibir tratamientos sistémicos o loco-regionales no curativos. Entre las opciones terapéuticas, la más empleada en estos pacientes es la embolización arterial sola o asociada con quimioterapia usando doxorrubicina o cisplatino (QETA). Si bien los estudios iniciales no demostraron beneficio con este tratamiento ${ }^{31}$, un metaanálisis reciente permite concluir que la QETA aumenta la supervivencia ${ }^{32}$. La quimioterapia por vía sistémica en pacientes con $\mathrm{CHC}$ es poco eficaz y por el momento no se dispone de un tratamiento estándar. La administración selectiva en la arteria de agentes quimioterápicos mezclados o no con lipiodol, sin oclusión arterial, no aumenta la eficacia, no reduce la toxicidad ni mejora el pronóstico.

En este grupo de pacientes, todavía mucho más numeroso que aquellos que se diagnostican en estadio precoz, pues supone aproximadamente un $70 \%$ de todos los $\mathrm{CHC}$, es necesaria la investigación de nuevas terapias.

\section{Evaluación de la respuesta}

En los pacientes sometidos a cirugía o a trasplante hepático no cabe un análisis de la respuesta mediante pruebas de imagen; la eficacia se valora en el estudio de la pieza de resección (satelitosis, invasión vascular, bordes de resección libres o no de tumor, etc) o en el explante. En cambio, en los pacientes sometidos a tratamientos loco-regionales, siguen objetivándose la lesión o lesiones previas al tratamiento, pero que cambian su comportamiento por la necrosis. En estos casos la eficacia de la respuesta local se suele definir según los criterios de la OMS, que mostramos en la Tabla VI².

TABLA VI

\section{Evaluación de la respuesta al tratamiento loco-regional} en el carcinoma hepatocelular

1. Respuesta completa (RC). Desaparición de la enfermedad y ausencia de nuevas lesiones determinada mediante dos observaciones no menos de 4 semanas entre ambas;

2. Respuesta parcial (RP). Reducción $>50 \%$ en el volumen tumoral total de todas las lesiones medibles determinado mediante dos observaciones con no menos de 4 semanas de intervalo entre las mismas;

3. Enfermedad estable (EE). Aquélla que no cumple criterios ni de $\mathrm{RC}$, ni de RP ni de enfermedad progresiva;

4. Enfermedad progresiva (EP). Incremento $>25 \%$ en el tamaño de uno o más lesiones medibles o la aparición de nuevas lesiones.

Para valorar la respuesta no basta con la medida bidimensional de los diámetros, puesto que no tiene en cuenta la necrosis tumoral por el tratamiento, ya que una necrosis extensa no va a acompañada por una reducción evidente en el diámetro de la lesión, por lo que es preciso es estimar la reducción en el volumen de tumor viable, que se reconoce mediante la ausencia de captación en la TC (33). En aproximadamente un $10-30 \%$ de los pacientes, la TC, que se suele realizar unas 4 semanas post-tratamiento muestra ausencia de captación (RC), y sin embargo en controles sucesivos se objetiva recidiva, estos casos se deben reclasificar como fracaso terapéutico. 


\section{Bibliografía}

1. Bosch FX, Ribes J, Borras J. Epidemiology of primary liver cancer. Semin Liver Dis 1999; 19:271-85.

2. Bruix J, Sherman $M$, Llovet JM, Beaugrand $M$, Lencioni $R$, Christensen $E$, Burroughs $A$, et al. Clinical management on hepatocellular carcinoma. Conclusions of the Barcelona-2000 EASL Conference. J Hepatol 2001; 35:421-30.

3. Velázquez RF, Rodríguez $M$, Navascues CA, Linares A, Pérez R, Sotorrios NG, Martínez I, Rodrigo L. Prospective analysis of risk factors for hepatocellular carcinoma in patients with liver cirrhosis. Hepatology. 2003; 37:520-7.

4. Collier J, Sherman M. Screening for hepatocellular carcinoma. Hepatology 1998; 27:273-8.

5. Barbara L, Benzi G, Gaiani S, Fusconi F, Zironi G, Siringo S et al. Natural history of small untreated hepatocellular carcinoma in cirrhosis: a multivariate analysis of prognostic factors of tumor growth rate and patient survival. Hepatology 1992; 16:132-7.

6. Sherman M. Alphafetoprotein: an obituary. J Hepatol. 2001; 34:603-5.

7. Bennett GL, Krinsky GA, Abitbol RJ, Kim SY, Theise ND, Teperman LW. Sonographic detection of hepatocellular carcinoma and dysplastic nodules in cirrhosis: correlation of pretransplantation sonography and liver explant pathology in 200 Patients. AJR 2002; 179:75-80.

8. Bhartia B, Ward J, Guthrie JA, Robinson PJ. Hepatocellular carcinoma in cirrhotic livers: double-contrast thin-section MR imaging with pathologic correlation of explanted tissue. AJR 2003; 180:577-84.

9. Teefey SA, Hildeboldt CC, Dehdashti F, Siegel BA, Peters MG, Heiken JP, Brown JJ, et al. Detection of primary hepatic malignancy in liver transplant candidates: prospective comparison of CT, MR imaging, US, and PET. Radiology 2003; 226:533-42.

10. Peterson MS, Baron RL, Marsh JW Jr, Oliver JH III, Confer SR, Hunt LE. Pretransplantation surveillance for possible hepatocellular carcinoma in patients with cirrhosis: epidemiology and CT-based tumor detection rate in 430 cases with surgical pathologic correlation. Radiology 2000; 217:743-9.

11. Burrel M, Llovet JM, Ayuso C, Iglesias C, Sala M, Miquel R, Caralt T, Ayuso JR, Sole M,3 Sanchez M, Bru C, Bruix J. MRI angiography is superior to helical CT for detection of HCC prior to liver transplantation: an explant correlation. Hepatology 2003; 38: 1034-42.

12. Mazzaferro V, Regalia E, Doci R, Andreola S, Pulvirenti A, Bozzetti F, Montalto F, et al. Liver transplantation for the treatment of small hepatocellular carcinomas in patients with cirrhosis. N Engl J Med 1996; 334:693-9.

13. Yao FY, Ferrell L, Bass NM, Watson JJ, Bacchetti P, Venook A, Ascher NL, Roberts JP. Liver transplantation for hepatocellular carcinoma: expansion of the tumor size limits does not adversely impact survival. Hepatology. 2001; 33:1394-403.

14. Sorensen JB, Klee M, Palshof T, Hansen HH. Performance status assessment in cancer patients. An inter-observer variability study. Br J Cancer 1993; 67:773-5.

15. Child CG, Turcotte JG: Surgery and portal hypertension. In: The liver and portal hypertension (Edited by: Child CG). Philadelphia, Saunders 1964, 50-64.

16. Pugh RNH, Murray-Lyon IM, Dawson JL, Pietroni MC, Williams $\mathrm{R}$ : Transection of the esophagus in bleeding oesophageal varices. Br J Surg 1973, 60:648-52.
17. Okuda K, Ohtsuki T, Obata H, Tomimatsu M, Okazaki N, Hasegawa H, Nakajima Y, Ohnishi K. Natural history of hepatocellular carcinoma and prognosis in relation to treatment. Study of 850 patients. Cancer 1985; 56:918-28.

18. The Cancer of the Liver Italian Program (CLIP) investigators. A new prognostic system for hepatocellular carcinoma: a retrospective study of 435 patiens. Hepatology 1998; 28:751-5.

19. The Cancer of the Liver Italian Program (CLIP) Investigators . Prospective validation of the CLIP score: a new prognostic system for patients with cirrhosis and hepatocellular carcinoma. Hepatology 2000; 31:840-5.

20. Izumi R, Shimizu K, li T, Yagi M, Matsui O, Nonomura A, Miyazaki I. Prognostic factors of hepatocellular carcinoma in patients undergoing hepatic resection. Gastroenterology 1994; 106:720-7.

21. Llovet JM, Bru C, Bruix J. Prognosis of hepatocellular carcinoma: the BCLC staging classification. Semin Liver Dis 1999; 19:329-38.

22. Llovet JM, Fuster J, Bruix J, Intention-to-treat analysis of surgical treatment for early hepatocellular carcinoma: resection versus transplantation, Hepatology 1999; 30:1434-40.

23. Livraghi T, Giorgio A, Marin G, Salmi A, de Sio I, Bolondi L et al. Hepatocellular carcinoma and cirrhosis in 746 patients: long-term results of percutaneous ethanol injection. Radiology 1995; 197:101-8.

24. Vilana R, Bruix J, Bru C, Ayuso C, Sole M, J. Rodes J. Tumor size determines the efficacy of percutaneous ethanol injection for the treatment of small hepatocellular carcinoma. Hepatology 1992; 16:353-7.

25. Castellano L, Calandra M, Del Vecchio-Blanco C, de Sio I, Predictive factors of survival and intrahepatic recurrence of hepatocellular carcinoma in cirrhosis after percutaneous ethanol injection: analysis of 71 patients. J Hepatol 1997; 27:862-70,

26. Lencioni R, Paolicchi A, Moretti M, Pinto F, Armillotta N, Di Giulio $M$ et al. Combined transcatheter arterial chemoembolization and percutaneous ethanol injection for the treatment of large hepatocellular carcinoma: local therapeutic effect and long-term survival rate. Eur Radiol 1998; 8:439-44.

27. De Baere T, Risse O, Kuoch V, Dromain C, Sengel C, Smayra T, Gamal El, Din M, Letoublon C, Elias D. Adverse events during radiofrequency treatment of 582 hepatic tumors. AJR 2003; 181:695-700.

28. Radiofrequency thermal ablation versus other interventions for hepatocellular carcinoma (Cochrane Review). The Cochrane Library, Issue 1, 2004.

29. Bruix J, Llovet JM. Prognostic prediction and treatment strategy in hepatocellular carcinoma. Hepatology 2002; 35:519-24.

30. Castells A, Bruix J, Bru C, Fuster J, Vilana R, Navasa M et al. Treatment of small hepatocellular carcinoma in cirrhotic patients: a cohort study comparing surgical resection and percutaneous ethanol injection, Hepatology 1993; 18:1121-6.

31. Bruix J, Llovet JM, Castells A, Montana X, Bru C, Ayuso MC et al. Transarterial embolization versus symptomatic treatment in patients with advanced hepatocellular carcinoma: results of a randomized, controlled trial in a single institution, Hepatology 1998; 27:1578-83.

32. Llovet JM, Bruix J. Systematic review of randomized trials for unresectable hepatocellular carcinoma: Chemoembolization improves survival. Hepatology 2003; 37:429-42.

33. Bartolozzi C, Cioni D, Donati F, Granai G, Lencioni R. Imaging evaluation of tumor response, In: C. Bartolozzi and R. Lencioni, eds., Liver malignancies. Diagnostic and interventional radiology, (1 st ed., edition, Springler-Verlag), 1999: 467-487. 